\title{
Laser-induced gas plasma etching of fused silica under ambient conditions
}

S. Elhadj, G. Guss, M. Matthews, I. Bass

February 14, 2013

SPIE Laser Damage 2012

Boulder, CO, United States

September 22, 2012 through September 25, 2012 
This document was prepared as an account of work sponsored by an agency of the United States government. Neither the United States government nor Lawrence Livermore National Security, LLC, nor any of their employees makes any warranty, expressed or implied, or assumes any legal liability or responsibility for the accuracy, completeness, or usefulness of any information, apparatus, product, or process disclosed, or represents that its use would not infringe privately owned rights. Reference herein to any specific commercial product, process, or service by trade name, trademark, manufacturer, or otherwise does not necessarily constitute or imply its endorsement, recommendation, or favoring by the United States government or Lawrence Livermore National Security, LLC. The views and opinions of authors expressed herein do not necessarily state or reflect those of the United States government or Lawrence Livermore National Security, LLC, and shall not be used for advertising or product endorsement purposes. 


\title{
Laser-induced gas plasma etching of fused silica under ambient conditions
}

\author{
Selim Elhadj*, Gabe Guss, Manyalibo J. Matthews, Isaac Bass \\ Lawrence Livermore National Laboratory, Livermore, California, 94550, USA
}

\begin{abstract}
Laser machining of optics to mitigate surface defects has greatly enhanced the ability to process large optics such as those found in fusion-class lasers. Recently, the use of assist reactive gases has shown promise in enhancing manifold etching rates relative to ambient conditions for CW-laser exposures. However, these methods still require significant heating of the substrate that induce residual stress, redeposit coverage, material flow, and compromise the final surface finish and damage threshold. While very reactive fluorinated gases are capable to reduce treatment temperatures even further, they are also inherently toxic and not readily transferable to large processing facilities. In this report, we look at whether a short-lived gas plasma could provide the safe and effective etchant sought, while still reducing the thermal load on the surface. We test this approach using a YAG laserinduced gas plasma to act as a source of the etchant for fused silica, a common optical material. The configuration and orientation of the beam and optical apparatus with respect to the surface was critical in preventing surface damage while etching the surface. Results with $\mathrm{N}_{2}$ and air gas plasmas are shown, along with a description of the various experimental implementations attempted.
\end{abstract}

Keywords: silica, gas plasma, laser, etching, heat, thermal, machining

*Correspondence: E-mail: elhadj2@llnl.gov; phone 1925 422-0270; fax 19254230792

\section{INTRODUCTION}

Laser based damage mitigation on silica optics has primarily been addressed by locally irradiating surfaces to remove damage sites, a process that leaves a pit on the surface from evaporation, ablation, and material flow. This approach has successfully been applied to prevent damage growth and re-initiation on fused silica optics in high power, fusion class lasers even under peak conditions. Careful pit shaping reduces beam modulation to levels below damage threshold [1], and also minimizes formation of caustic rim structures at the periphery of the pit. Continuous increases in both beam fluence and shot turnover rates increase the stringency of the damage mitigation strategies, which must subsequently evolve to address any new optical damage mechanism.

The formation of rims on laser treated surfaces is usually associated with displacement of melted silica by either Marangoni driven flow or from recoil pressure that push material from the center of the pit to the outside [2]. One damage mitigation approach focuses a moving beam of short $\mathrm{CO}_{2}$ laser pulses $(\sim \mu \mathrm{s})$ that progressively and locally ablates the material into the desired shape, while reducing heating of the substrate [3]. In spite of the high peak temperatures reached $\sim 4500 \mathrm{~K}$, this reduction in substrate heating is due to the short thermal diffusion lengths $\sim \sqrt{ } D t_{\text {exp }}$ from the short, but intense $\mu$ s exposures, where $D$ is the thermal diffusion and $t_{\text {exp }}$, the laser exposure time. Another, lower temperature approach $\sim 2000-3000 \mathrm{~K}$ uses a simpler protocol with a fixed $\mathrm{CW} \mathrm{CO}_{2}$ beam under steady state laser heating conditions ( $t_{\exp } \sim$ seconds) [4]. Drawbacks of these long laser exposures include significant bulk material heating, densification, residual stress, and large rims [5, 6], making this approach more problematic for routine damage mitigation. To address this issue, reactive assist gases have been applied to reduce the laser treatment temperature by about $\sim 250 \mathrm{~K}$ depending on the gas chemistry used [5]. That reduction in temperature 
increases melt viscosity while reducing thermocapillary flow and residual stress $[5,6]$. However, because fused silica is highly inert even under reactive gas atmospheres, high evaporation temperatures around $2800 \mathrm{~K}$ are still required to remove and flow enough material over practical time scales [5]. To lower the treatment temperature further below the glass transition point, extremely reactive gases, such as CF4, could potentially be used[7]. However these gases are toxic and difficult to handle in processing facilities designed for large aperture optics. Therefore an alternative approach, described here, was explored that uses a laser induced gas plasma to produce a reactive gas phase etchant for fused silica.

An intense beam can ignite a plasma in air with a high degree of ionization at the focus point of the laser [8]. The gas plasma can then displace or react with the silica surface to produce volatile species. Most of the laser energy can in principle be coupled into the gas while leaving the surface un-affected, except for the plasma etching and, hopefully, with a minimal thermal footprint to prevent rim formation. Furthermore, although the plasma gas is very reactive, it is also short-lived due to rapid ionic recombination at atmospheric pressures [9]. Thus, the source of the plasma should be chemically quenched well before propagating into the surrounding, remaining safe for use as a localized etching gas. In this study, the extent to which the plasma gas couples heat into the substrate is assessed from analysis of the final surface shape for signs of material flow, while etching is assessed from the depth of material removed. The challenge was to optically breakdown a gas (air and $\mathrm{N}_{2}$ ) with intense laser pulses to generate an etching plasma near the optics for treatment without damaging it. Also, whether $\mathrm{O}_{2}, \mathrm{~N}_{2}$, or ambient air plasma without fluorinated compounds- would breakdown into components reactive enough to etch silica was not clear [7, 10]. To that end, four different experimental configurations were attempted to produce the plasma near the silica optics. A front facing exposure, a back facing exposure, a front facing exposure with gas nozzle to displace the plasma closer to the surface and, finally, a glancing configuration where the beam comes in at a slight angle with the surface. The feasibility and effectiveness of the laser induced gas plasma etching of fused silica using these four configurations is presented here. 


\section{EXPERIMENTAL}

\section{1 laser-induced gas plasma configurations}

The setup used to generate laser induced gas plasma near the fused silica sample for treatment is shown in Fig. 1. In Fig. 1(A) a YAG laser beam is focused through a $100 \mathrm{~mm}$ fused silica lens and the plasma cloud formed at focus while propagating backward [11]. The fused silica Corning 7980 sample (50 mm diameter, $10 \mathrm{~mm}$ thick) is brought in proximity to the gas plasma with a mechanized stage. The apparent plasma location was made by visual inspection to within $\sim 0.5 \mathrm{~mm}$ and, sometimes, was driven well into the surface making apparent contact with the material. In Fig. 4(B) the same optical setup described above was used but the beam is focused through the back of the sample instead. This setup (B) was thought to be advantageous compared to (A) because the plasma would radiate backwards toward the surface and the lens, producing a more intimate contact with the surface while the local incoming laser fluence at the surface would be reduced. In Fig. 4(C) the sample is orientated at a glancing angle of about 15 degrees with respect to the beam axis. In this configuration, the sample could be moved away or closer to the surface by moving the stage vertical or parallel to the beam propagation axis due the slight tilt of the sample. Finally, Fig. 4(D) shows an in-house aluminum nozzle mounted with a focusing lens on the back side, which acts as both a window to seal the nozzle and to focus the beam within the nozzle chamber. To shield the sample from the direct beam exposure, the beam was propagated slightly of axis with respect to the nozzle opening (12.5 mm round). Compressed gases $\left(100 \% \mathrm{~N}_{2}\right.$ and air) were supplied to the nozzle through a mass flow controller capable of reaching flow rates up to $100 \mathrm{~L} / \mathrm{min}$. As indicated in Fig. 4(D) the laser beam was focused about $4 \mathrm{~mm}$ from the sample surface inside the nozzle, with $\sim 1 \mathrm{~mm}$ separation between the aperture and the sample.
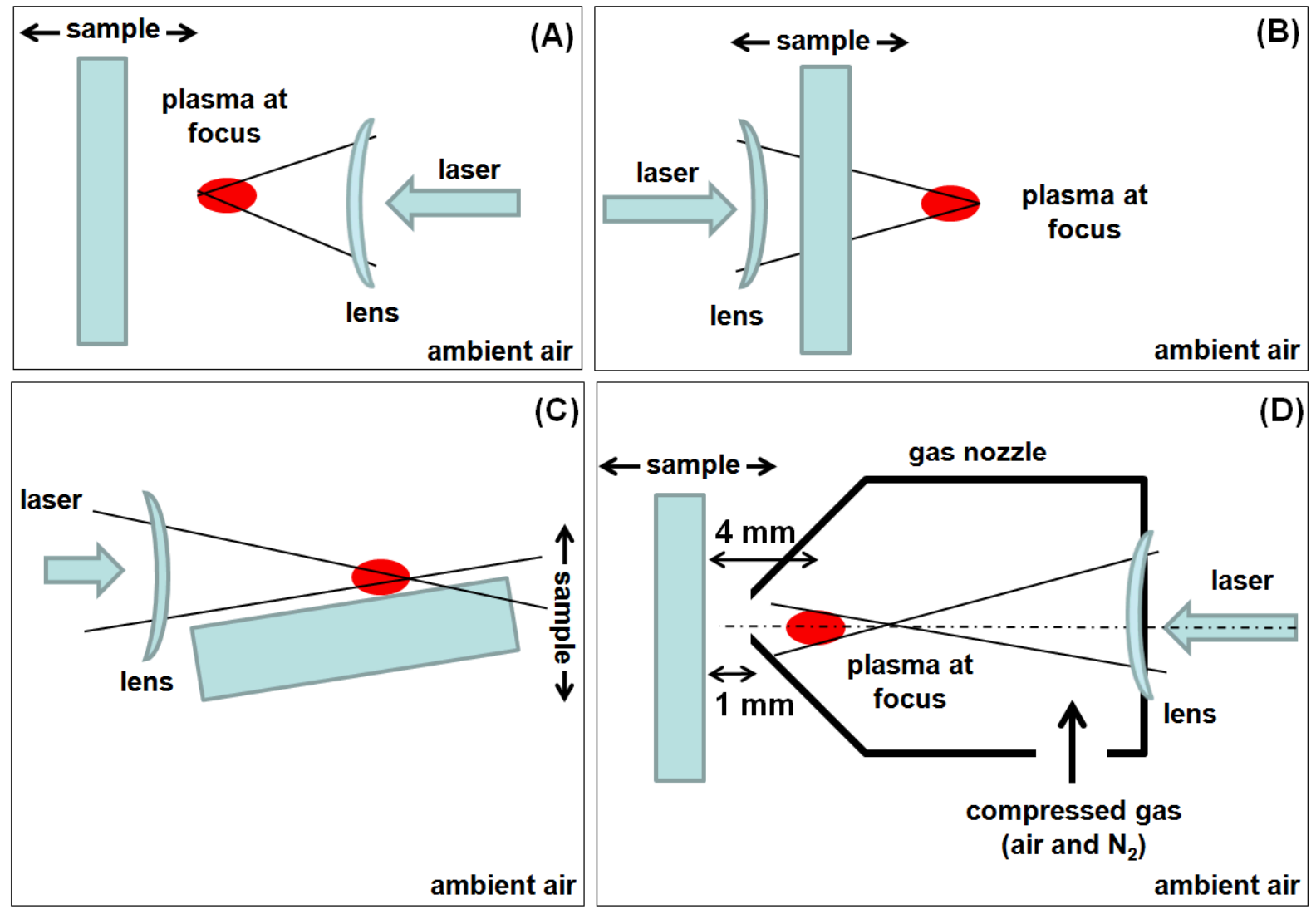

Figure 1. Schematics of the four configurations used to generate a laser induced gas plasma for etching a fused silica sample surface. In all four configurations shown, the sample is mounted on a 
mechanized stage to move the surface closer or away from the plasma cloud (filled ellipse), which remains fixed. (A) The pulsed YAG laser beam is focused facing the front of the sample near the surface. In (B) the beam is focused near the surface but through the backside of the sample. (C) The beam is focused at a glancing angle of about 15 degrees. (D) Same configuration as in (A), except a nozzle is used to supply compressed gases $\left(\mathrm{N}_{2}\right.$ and air) at flow rates up to $100 \mathrm{~L} / \mathrm{min}$ through a $12.5 \mathrm{~mm}$ nozzle aperture (not to scale). The beam is focused about $4 \mathrm{~mm}$ from the sample surface and aligned slightly of axis with the aperture to shield the front surface from the laser beam as shown. The plasma cloud is drawn in front of the focus lines in (D) to indicate the potential plasma displacement from the flow. The nozzle was positioned about $1 \mathrm{~mm}$ from the surface.

A picture of the gas plasma generated inside the nozzle appears bluish with some of the glow reflected on the surface of the sample as indicated by the dashed circle (Fig. 2)

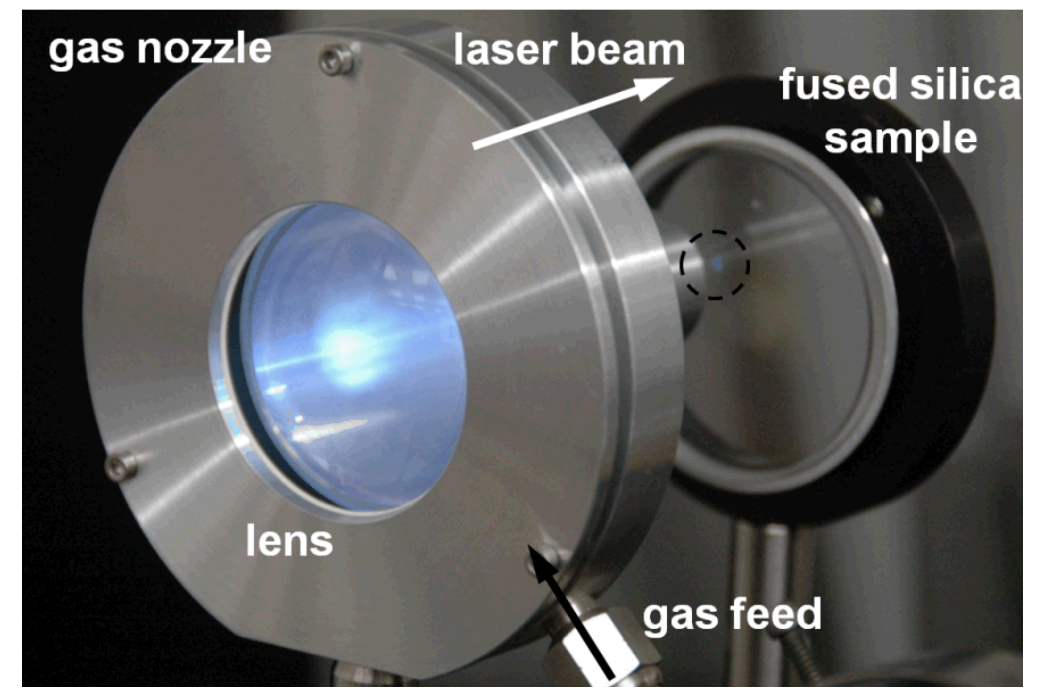

Figure 2. Picture of an in-house aluminum gas flow nozzle used in experiments generating laser induced gas plasma (bluish spark emissions shown). The plasma emissions appear to reflect (dotted circle) from the front side of the 2 inch round fused silica sample. A gas feed allows for controlled mass flow rates of up to $100 \mathrm{~L} / \mathrm{min}$ using a mass flow controller.

\section{2 laser setup for laser induced gas plasma}

A flash-lamp pumped SP Quanta Ray ND:YAG laser operating at $355 \mathrm{~nm}, 10 \mathrm{~Hz}$ repetition rate and $7.5 \mathrm{~ns}$ pulse length was focused to a tight spot through a $100 \mathrm{~mm}$ focal length lens. The estimated size of the beam waist was 10 $\mu \mathrm{m}$. The energy per pulse of the laser was adjusted using an external wave plate polarizer attenuator. During setup of the experiment, the energy was increased until plasma generation in air was observed. This energy is estimated to be $25 \mathrm{~mJ}$. The fused silica sample was then inserted in one of the described configurations and a number of sites on the sample were exposed to the plasma, with the time of exposure nominally 1 min except where indicated.

\subsection{Surface characterization}

Results of laser induced gas plasma exposures were characterize with a ZYGO white light interferometer (Zygo Corp., Middlefield, CT, USA) to measure surface profile features and determine pit depths. The vertical resolution of the instrument is on the order of $1 \mathrm{~nm}$ and the lateral resolution $\sim 1.0 \mu \mathrm{m}$. For deeper or rougher sites $>10 \mu \mathrm{m}$, high resolution 3D shapes were measured using a STIL probe chromatic confocal imaging system (STIL, 
Aix en Provence, France) with sub-micron vertical resolution, and 2-5 $\mu \mathrm{m}$ spatial resolution depending on the scanning settings. Optical imaging was used to monitor when laser damage occurred on the surface.

\section{RESULTS AND DISCUSSION}

\subsection{Front and back side configurations}

It became apparent that with the laser energy set at a minimum $(25 \mathrm{~mJ})$ to generate a gas plasma, the fused silica surface damaged within the first exposure, producing the typical ns UV damaged and fractured pits [12] illustrated in Fig. 3 below. Small particles of ejected material with scalded areas are apparent throughout the site and the glassy fractured pit. The occurrence of plasma scalding was particularly pronounced when the sample surface was brought close enough to make apparent contact with the gas induced plasma. When the sample was moved further than $\sim 2 \mathrm{~mm}$, the surface did not damage but no apparent changes to the surface occurred, including a lack of etching. When the laser passes through the sample in the back side configuration the sample damaged more easily due to the lower damage threshold of the exit surface $[13,14]$ that occurred in spite of the lower effective beam fluence at the surface. The gas plasma in the back side configuration can reflect the beam back towards the surface to cause damage. Conversely, in the front side configuration the gas plasma can shield some of the beam after plasma onset.

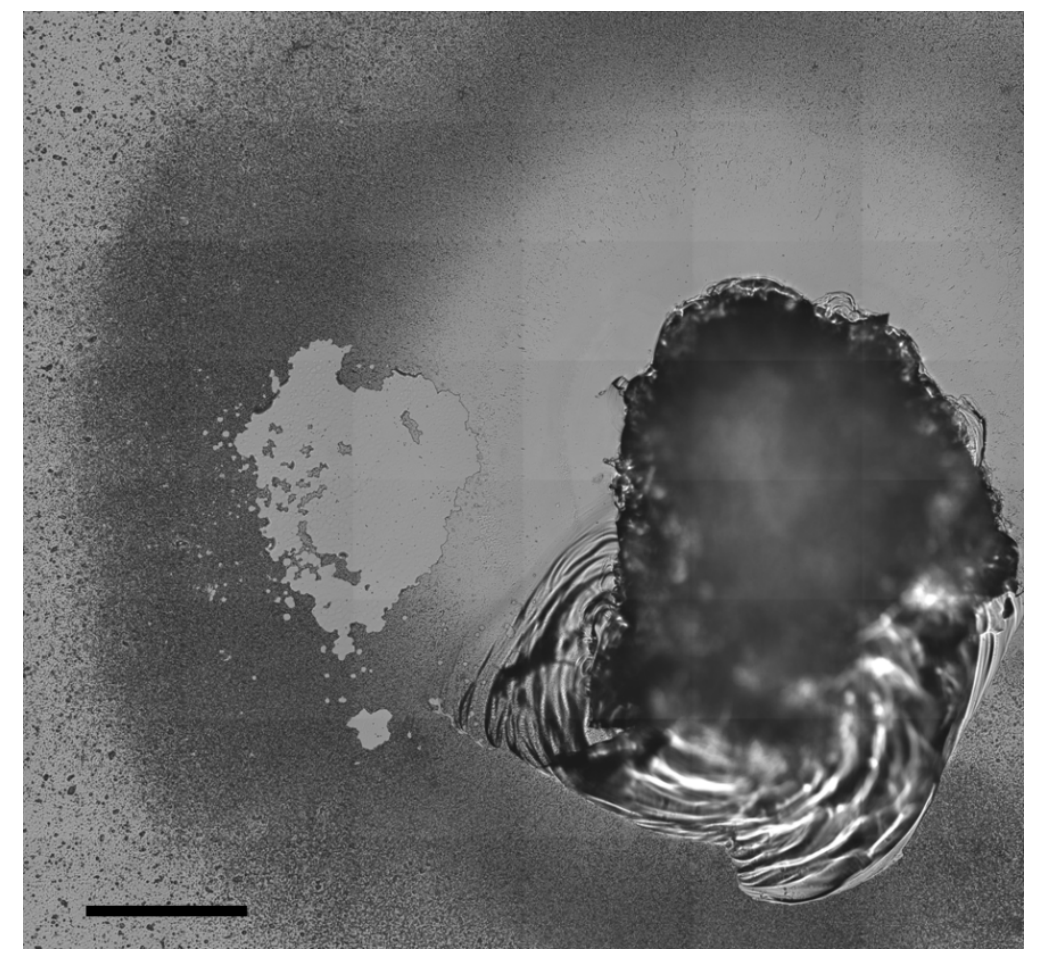

Figure 3. High resolution stitched images of a damage site resulting from UV laser exposure in the front side configuration of the laser induced gas plasma setup (Fig. 1). Scale bar represents 200 $\mu \mathrm{m}$.

\subsection{Front side configuration with nozzle flow}

By adding a gas flow nozzle to the front side configuration the sample surface could be shielded from the incoming beam to prevent the damaged that was observed (sec. 3.1). Although the beam was defocused when obstructed partially inside the nozzle, particulates appeared on the sample surface after $30 \mathrm{~min}$ of treatment (Fig. 4). These particulates were likely etched from the nozzle wall by the beam. After cleaning the surface of debris, no 
surface etching was apparent from white light interferometry profiling, indicating a lack of significant interaction between the gas plasma and the sample whether $\mathrm{N}_{2}$ or air was used. From the flow of the gas, the plasma could, in principle, be moved closer to the surface gas after it had ignited. Considering a superficial velocity out of the nozzle aperture of $\sim 4 \mathrm{~m} / \mathrm{s}$ at the maximum flow rate, the resulting time to traverse the $\sim 1 \mathrm{~mm}$ aperture-to-surface distance would be about $80 \mu \mathrm{s}$. This time is comparable to the reported plasma emissions decay times (1/e) of 10-70 $\mu \mathrm{s}$ produced from $40 \mathrm{~ns}$ pulsed-laser plasma explosions in ambient air [15]. Thus some of the plasma molecules and atoms could remain ionized on their way toward the surface. With a laser rep rate of $10 \mathrm{~Hz}\left(10^{5} \mu\right.$ s periods) these plasma gas pulses likely do not interact with each other and with successive laser pulses. Nevertheless, the plasma cloud did not appear to be moving forward, whether gas flow was applied or not in the nozzle. The reason may be that the cloud is produced as an expanding high pressure plasma that can oppose displacement from the flowing gas.

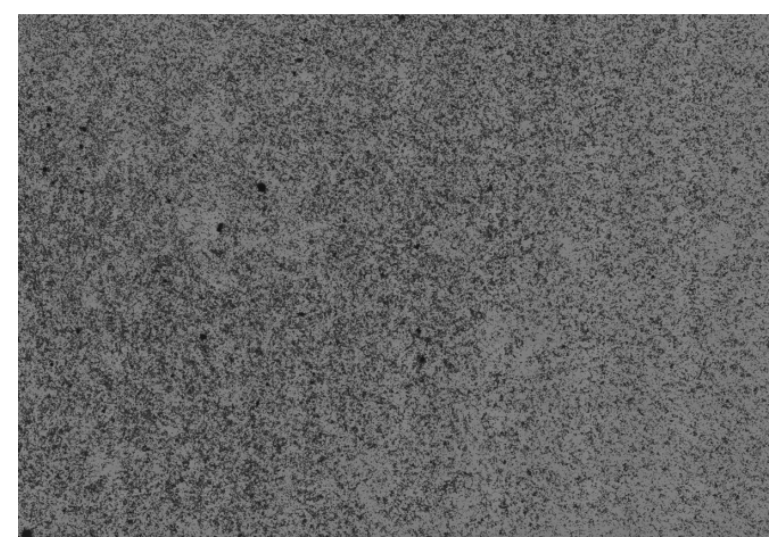

Figure 4. Microscopy image of the sample surface following laser-induced gas plasma inside the gas flow nozzle (Fig. 2). Micron size particles from the nozzle walls appeared sprayed on the surface.

\subsection{Glancing configuration}

All three previous configurations either failed to produce any etching, or damaged before the laser induced gas plasma could affect the surface. In contrast, the fourth configuration attempted with the laser beam coming at a glancing angle did not produce any damage under the same laser pulse breakdown energies, yet managed to generate pits of micron-sized depths with a $1 \mathrm{~min}$ beam dwell time depending on how close the plasma was to the surface (Fig. 5). However, the surface shows evidence of melting and 5-10 nm rim formation indicating that significant surface heating resulted from the gas plasma application. Since the surface appears to lack large areas of recondensed materials, the temperature of the surface must have remained well below the boiling point of silica $(\sim 2800 \mathrm{~K})[16]$. Still, the surface temperature must also have been higher than the working point of fused silica $(\sim 2300 \mathrm{~K})$ to produce melt flow and rim formation [5]. Thus we estimate that the effective surface temperature during plasma application in the beam glancing configuration to be approximately between $2300 \mathrm{~K}$ and $2800 \mathrm{~K}$. 


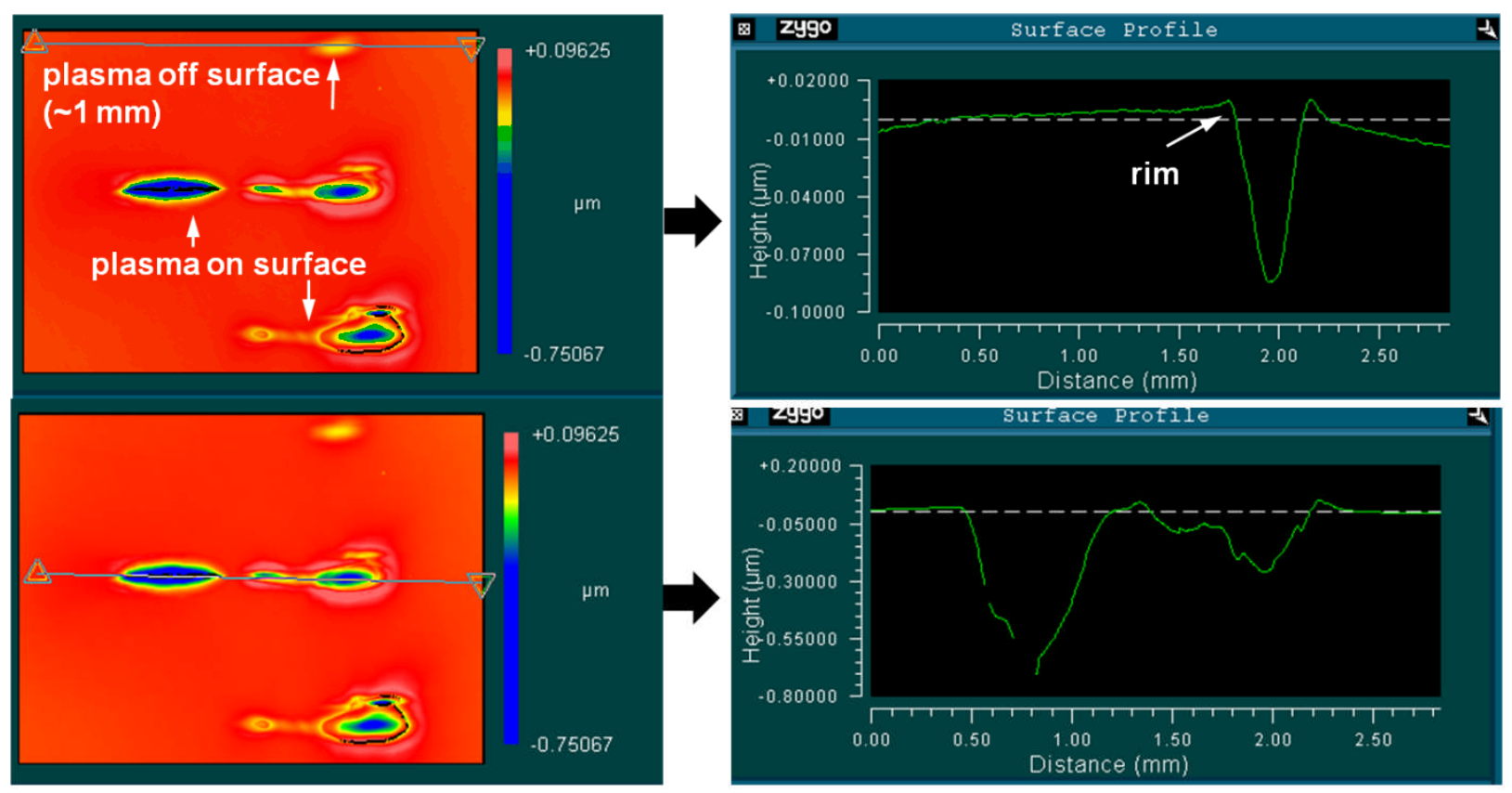

Figure 5. White light interferometry images of a fused silica surface treated with a gas plasma using the beam glancing configuration (Fig.1). Surface depths are derived along the line profiles indicated on the surface height images.

Linear trenches on the surface could also be produced by simply translating the stage laterally (Fig. 6) while maintaining the gas plasma in contact with the surface. The process produces a somewhat even depth profiles, although the trench extends laterally according to the gas plasma foot print as depicted in Fig. 7. 


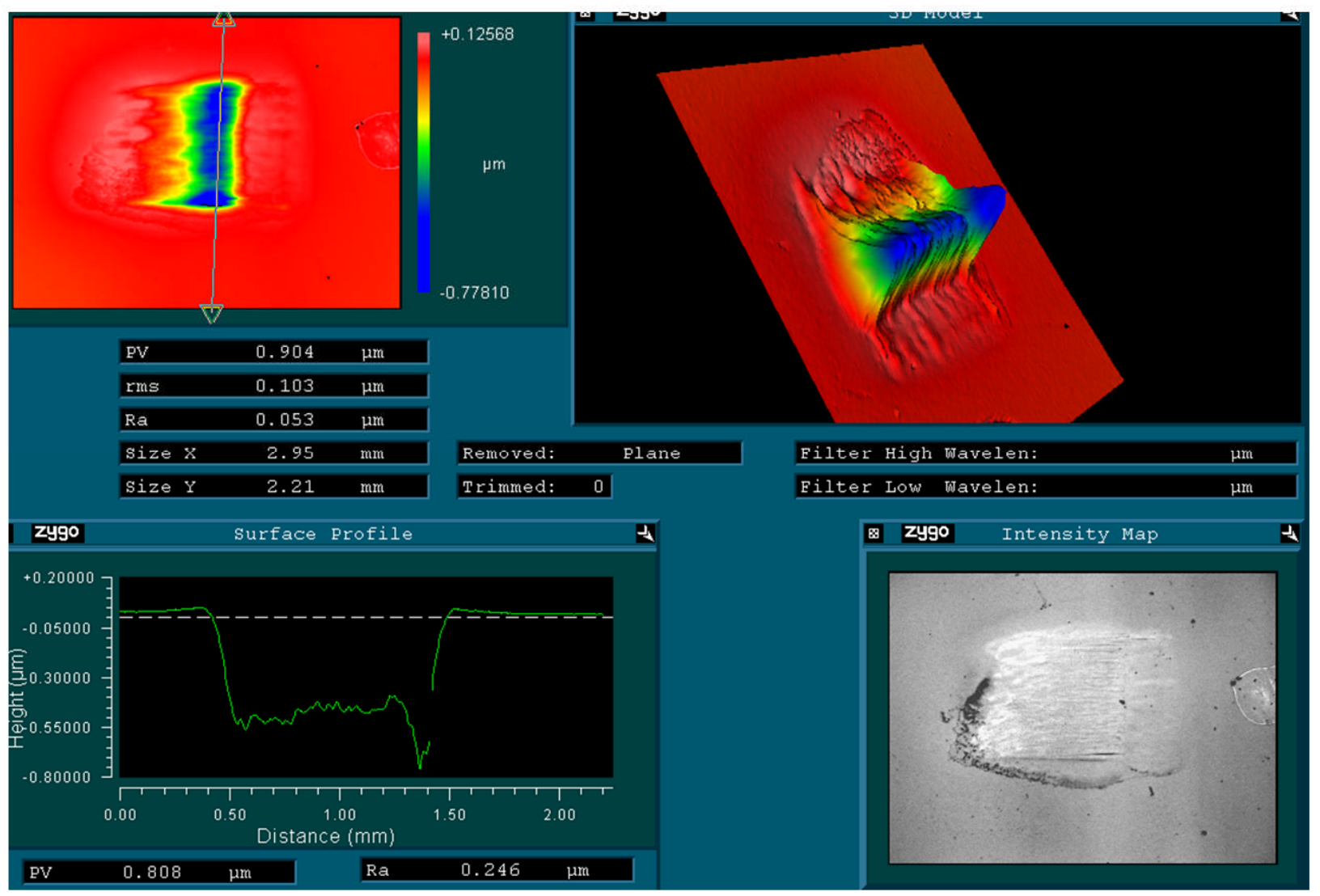

Figure 6. White light interferometry images of a fused silica surface treated with a gas plasma using the beam glancing configuration (Fig.1). The stage was moved laterally $\sim 1$ $\mathrm{mm}$ to produce a linear trench. Surface depths are derived along the line profiles indicated on the surface height images. The bottom right image shows the corresponding intensity map of the trench, while above it is the 3D inverted height map showing the underlying structure of the trench.

In Fig. 7, a deep $(\sim 30 \mu \mathrm{m})$ feature was created from the laser-induced gas plasma at a glancing angle. The surface appears relatively smooth with a melted splash region around it, but no apparent fracturing that would be expected from damage with ns UV pulses [12]. The general shape of the feature is consistent with previous observations of the gas plasma spark produced under conditions similar those in this study [17]. The spark expands more in the axial direction (laser propagation direction) than in the lateral directions for $\sim 5 \mu$ s after ignition, producing the elongated shape (Fig. 7). Even the slightly lobed features apparent in Fig. 7(B) are reminiscent of the reported glow of the two-lobed intensity patterns from time gate ICCD camera imaging [11]. Lastly, the melted region extends well beyond $\sim 300 \mu \mathrm{m}$ the $<150 \mu \mathrm{m}$ wide evaporated pit, with a slight roughening of surface at the edges (near the dotted lines). This very distinct and shallow $(<0.5 \mu \mathrm{m})$ area of melted silica is likely the result of the exposure to the plasma thermal emissions; the roughening at the edges could be produced from the high pressure gradients that exist at plasma/air interfaces that push the melt outward into small rims [18]. 


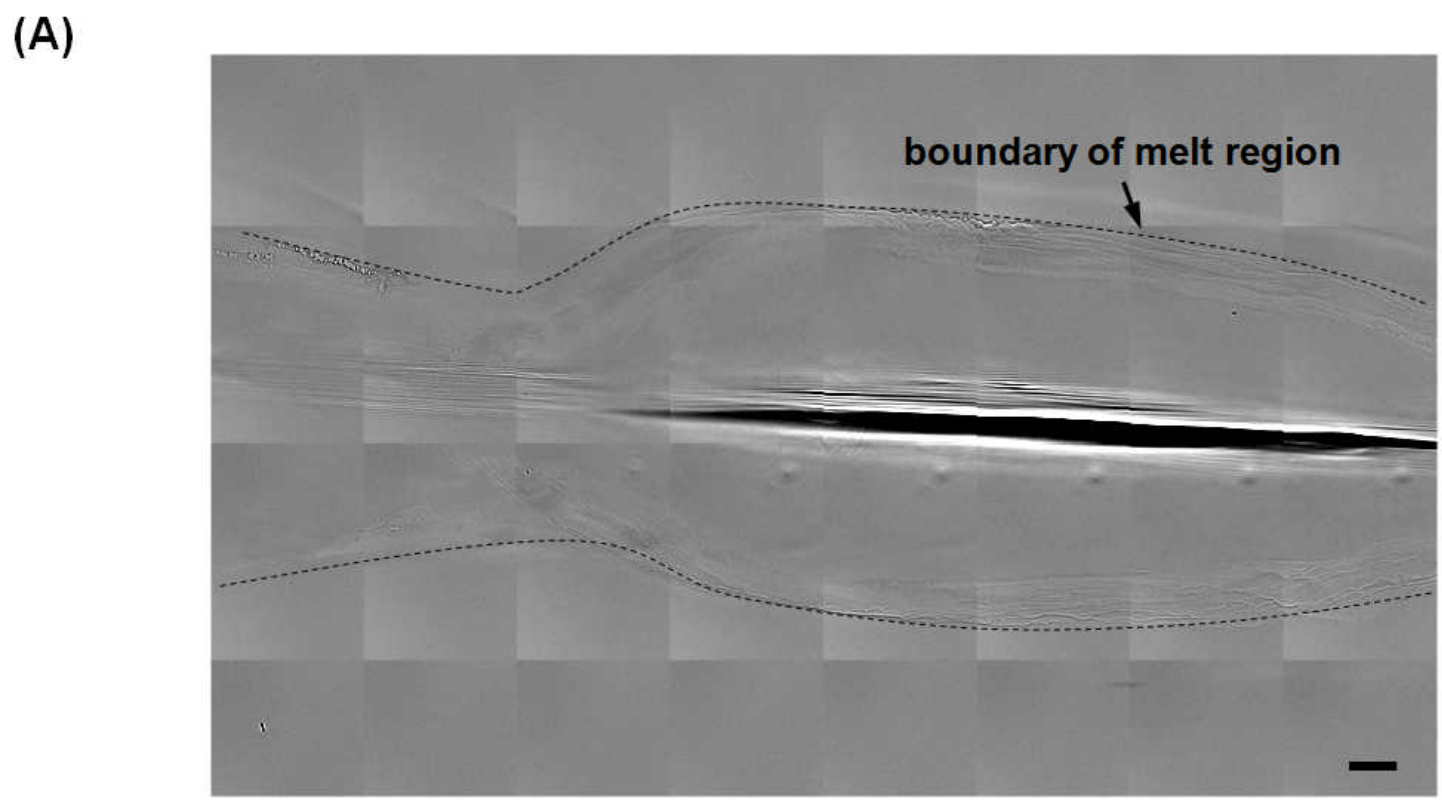

(B)

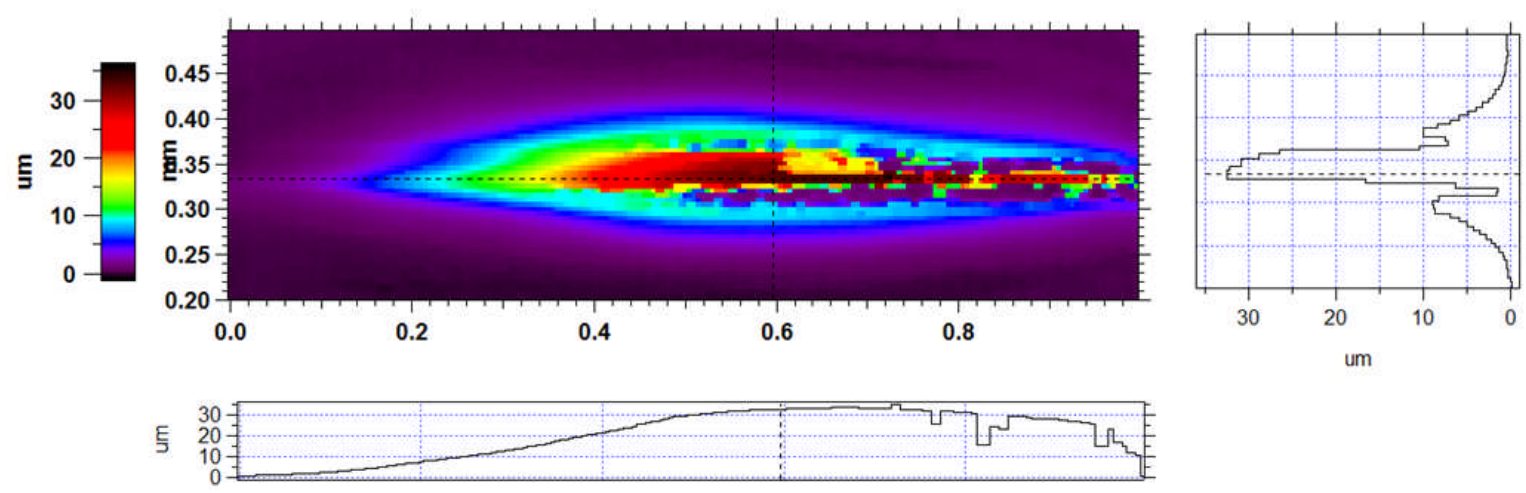

Fig. 7 (A) High resolution stitched images of a gas plasma etched site resulting from laser exposure in the glancing configuration setup (Fig. 1). This etched site was produced with a fixed stage and a fixed beam propagating from the left of the image to the right parallel to a tilted surface (scale bar represents $50 \mu \mathrm{m}$ ). The apparent surface melted region is delineated with the dotted line to guide the eye. (B) STIL probe height map and depth profiles of the site shown in (A).

\section{CONCLUSIONS}

We describe a feasibility study of fused silica etching using laser-induced gas plasma interactions. The goal was to use the reactive gas plasma to minimize the thermal coupling to the surface and prevent surface deformation and, in particular, rim formation. Four different optical configurations were tested, all with a $7.5 \mathrm{~ns}$ UV YAG laser at $10 \mathrm{~Hz}$ repetition rate and $1 \mathrm{~min}$ treatment time. Only the glancing beam configuration produced some etching but mostly from heating and vaporizing the sample. The heating was discernible from the typical rim structures produced from melt flow above the working point of fused silica. We estimated that surface temperatures to be between $2300 \mathrm{~K}$ and $2800 \mathrm{~K}$. Two other configurations with laser beam in the light of sight of the sample immediately damaged the surface because of the direct exposure to the beam when bringing the gas plasma close enough to the surface to interact. In the same front facing configuration but with an added gas nozzle flow in the system, the beam was partially screened preventing sample damage, albeit without producing any measureable 
surface etching. In general the reasons for this lack of apparent effect of the laser induced gas plasma could be attributed to the short plasma lifetime at atmospheric pressures or a lack of reactivity of the plasma source gases used with silica $\left(\mathrm{N}_{2}\right.$, air, and ambient air). Whether it is possible that the gas plasma could be cooled enough during its expansion, while the plasma molecules remain reactive is not clear from this study, which mostly focused on the gross and microstructural changes. Future time gated spectroscopic measurement could help in addressing those questions. Potential improvements to the experimental system include use of fluorinated gas if tolerable, a more precise diagnostic to locate the plasma cloud near the surface, and operation at lower pressures to reduce the plasma recombination rates and extend its lifetime. Finally, the nozzle-based approach appears more practical to apply the plasma gas. However, issues of the beam interacting with the nozzle wall need to be accounted for in the design to prevent long term degradation.

\section{ACKNOWLEDGMENTS}

This work performed under the auspices of the U.S. Department of Energy by Lawrence Livermore National Laboratory under Contract DE-AC52-07NA27344.

\section{REFERENCES}

[1] M. J. Matthews, I. L. Bass, G. M. Guss et al., "Downstream intensification effects associated with CO2 laser mitigation of fused silica - art. no. 67200A." 6720, 67200A.

[2] J. Mazumder, "Overview of Melt Dynamics in Laser Processing," Optical Engineering, 30(8), 1208-1219 (1991).

[3] I. L. Bass, G. M. Guss, M. J. Nostrand et al., "An Improved Method of Mitigating Laser Induced Surface Damage Growth in Fused Silica Using a Rastered, Pulsed CO2 Laser." 7842, 784220.

[4] J. J. Adams, M. Bolourchi, J. D. Bude et al., "Results of applying a non-evaporative mitigation technique to laser-initiated surface damage on fused-silica," Laser-Induced Damage in Optical Materials: 2010, 7842, (2010).

[5] S. Elhadj, M. J. Matthews, G. M. Guss et al., "Laser-based dynamic evaporation and surface shaping of fused silica with assist gases: a path to rimless laser machining," Applied Physics B: Lasers and Optics, (in print), (2012).

[6] R. M. Vignes, T. F. Soules, J. S. Stolken et al., "Thermo-mechanical modeling of laser-induced structural relaxation and deformation of $\mathrm{SiO} 2$ glass," Journal of the American Ceramic Society, (in print), (2012).

[7] K. R. Williams, and R. S. Muller, "Etch rates for micromachining processing," Journal of Microelectromechanical Systems, 5(4), 256-269 (1996).

[8] H. Conrads, and M. Schmidt, "Plasma generation and plasma sources," Plasma Sources Science \& Technology, 9(4), 441-454 (2000).

[9] R. J. Vidmar, "On the Use of Atmospheric-Pressure Plasmas as Electromagnetic Reflectors and Absorbers," leee Transactions on Plasma Science, 18(4), 733-741 (1990).

[10] K. R. Williams, K. Gupta, and M. Wasilik, "Etch rates for micromachining processing - Part II," Journal of Microelectromechanical Systems, 12(6), 761-778 (2003).

[11] C. V. Bindhu, S. S. Harilal, M. S. Tillack et al., "Energy absorption and propagation in lasercreated sparks," Applied Spectroscopy, 58(6), 719-726 (2004).

[12] R. A. Negres, M. A. Norton, D. A. Cross et al., "Growth behavior of laser-induced damage on fused silica optics under UV, ns laser irradiation," Optics Express, 18(19), 19966-19976 (2010).

[13] N. L. Boling, M. D. Crisp, and G. Dube, "Laser-Induced Surface Damage," Applied Optics, 12(4), 650-660 (1973). 
[14] M. A. Norton, E. E. Donohue, M. D. Feit et al., "Growth of laser damage on the input surface of SiO2 at 351 nm - art. no. 64030L," Laser-Induced Damage in Optical Materials: 2006, 6403, L4030-L4030 (2007).

[15] S. Brieschenk, R. Hruschka, S. O'Byrne et al., "High-speed time-resolved visualisation of laserinduced plasma explosions," 28th International Congress on High-Speed Imaging and Photonics, 7126, (2009).

[16] H. L. Schick, "A Thermodynamic Analysis of the High-Temperature Vaporization Properties of Silica," Chemical Reviews, 60(4), 331-362 (1960).

[17] S. S. Harital, and B. Harilal, Diagnostics of laser induced spark in air using fast ICCD photography (Technical report). Fusion Division, Center for Energy Research, University of California, San Diego La Jolla, CA (2002).

[18] A. Ben-Yakar, R. L. Byer, A. Harkin et al., "Morphology of femtosecond-laser-ablated borosilicate glass surfaces," Applied Physics Letters, 83(15), 3030-3032 (2003). 